\title{
Light-scattering characterization of transparent substrates
}

\author{
Myriam Zerrad, Carole Deumié, Michel Lequime, Claude Amra, and Mike Ewart
}

\begin{abstract}
Angle-resolved light scattering has been used for decades to quantify the surface roughness of optical components. However, because this technique is affected by the contribution of both interfaces of the sample, it cannot be applied to transparent substrates. We show how to overcome this issue and apply these principles to the characterization of superpolished samples. (C) 2006 Optical Society of America

OCIS codes: $120.5820,120.6660$.
\end{abstract}

\section{Introduction}

Far-field light scattering has been extensively studied these past decades ${ }^{1-10}$ as a technique for surfaceroughness characterization. In the case of opaque samples, using both angle-resolved-scattering (ARS) measurements and a specific electromagnetic theory, we can deduce the surface-roughness spectrum, which once integrated provides a root mean square of the roughness in the optical bandwidth. Roughness values can currently be measured down to $0.1 \AA$, with a relative accuracy of approximately $3 \%$.

However, this technique is valid mainly in the case of opaque substrates. When the substrates are transparent, scattering from both interfaces is simultaneously collected by the receiver in the far field, so that additional discrimination is required.11,12 In this paper we present the modifications we performed to the experimental setup, which are based on geometrical considerations, to overcome this issue.

\section{Characterization of Opaque Substrates}

For a single surface whose irregularities are assumed to be much smaller than the illumination wavelength, a simple relationship issued from first-order theory ${ }^{1,2,6,13-15}$ allows us to determine the surface

M. Zerrad (myriam.zerrad@fresnel.fr), C. Deumié, M. Lequime, and C. Amra are with Institut Fresnel, Unité Mixte de RechercheCNRSTIC 6133, Ecole Généraliste d'Ingénieurs de Marseille, Université Paul Cézanne Aix-Marseille I, Domaine Universitaire de St. Jérôme, 13397 Marseille Cedex 20, France. M. Ewart is with WZW Optic AG, Postfach 42, Mühlesteinstrasse 12, 9436 Balgach, Switzerland.

Received 1 March 2005; revised 25 July 2005; accepted $13 \mathrm{Au}-$ gust 2005 .

0003-6935/06/071402-08 $\$ 15.00 / 0$

C 2006 Optical Society of America properties from the angular data of the scattering pattern:

$$
I(\theta, \phi)=C^{ \pm}(\theta, \phi) \gamma(\theta, \phi),
$$

where $\theta$ and $\phi$ are the normal and polar angles, respectively, that describe a scattering direction in the far field (as illustrated in Fig. 1) and $C^{ \pm}(\theta, \phi)$ is an optical factor that takes into account the material index, polarization, and wavelength. $C^{ \pm}$is defined for normal illumination and natural light in reflection $\left(C^{-}\right)$or in transmission $\left(C^{+}\right)$by

$$
\begin{aligned}
& C^{-}(\theta, \phi)=C^{-}(\theta)=\frac{1}{2}\left(\frac{2 \pi n_{0}}{\lambda}\right)^{2}\left[\cos ^{2} \theta_{0}\left|q_{s}\right|^{2}+\left|q_{p}\right|^{2}\right], \\
& C^{+}(\theta, \phi)=\frac{1}{2}\left(\frac{n_{s}^{3}}{n_{0}}\right)\left(\frac{2 \pi}{\lambda}\right)^{2}\left[\cos ^{2} \theta_{s}\left|q_{s}\right|^{2}+\left|q_{p}\right|^{2}\right],
\end{aligned}
$$

with

$$
\begin{aligned}
q_{s}(\theta)= & j\left(\frac{2 \pi}{\lambda}\right) 2 n_{0}\left(n_{0}-n_{s}\right)\left(n_{0} \cos \theta_{0}\right. \\
& \left.+n_{s} \cos \theta_{s}\right)^{-1} \\
q_{p}(\theta)= & j\left(\frac{2 \pi}{\lambda}\right) 2 n_{0}\left(n_{0}-n_{s}\right)\left(n_{0} / \cos \theta_{0}\right. \\
& \left.+n_{s} / \cos \theta_{s}\right)^{-1} \\
n_{0} \sin \theta_{0}= & n_{s} \sin \theta_{s} .
\end{aligned}
$$

The roughness spectrum $\gamma(\theta, \phi)$ of the surface is 


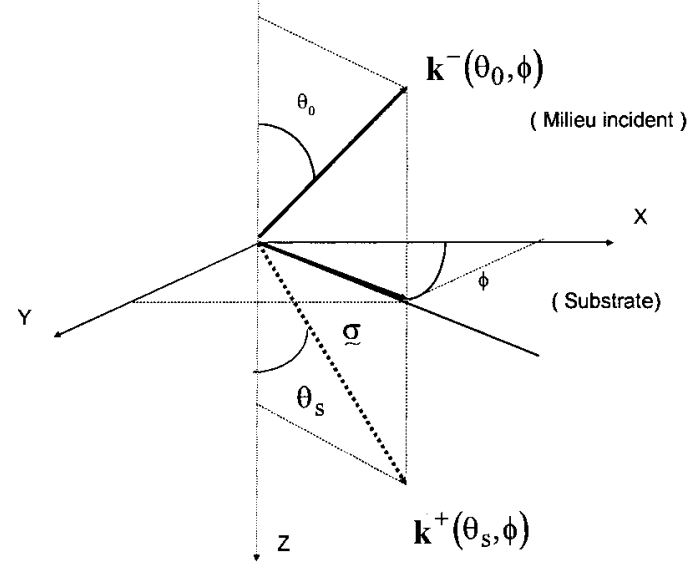

Fig. 1. Scattering angles.

given by

$$
\gamma=\frac{4 \pi^{2}}{S}|\hat{h}(\boldsymbol{\sigma})|^{2}
$$

where $\hat{h}$ is the Fourier transform of the surface profile $h(\mathbf{r})=h(x, y) ; S$ is the illuminated area on the sample, and $\boldsymbol{\sigma}=2 \pi(\sin \theta / \lambda)\{\cos \phi ; \sin \phi\}=2 \pi v$, where $v$ is the spatial frequency. With Eq. (1) the spectrum can be extracted from measurements, which allows us to quantify the roughness value $\delta$, or root mean square, in the optical bandwidth:

$$
\delta^{2}=\frac{1}{S} \int_{s} h^{2}(\mathbf{r}) \mathrm{d} \mathbf{r}=\int_{\boldsymbol{\sigma}} \gamma(\boldsymbol{\sigma}) \mathrm{d} \boldsymbol{\sigma},
$$

with $\mathrm{d} \mathbf{r}=\mathrm{d} x \mathrm{~d} y$ and $\mathrm{d} \boldsymbol{\sigma}=\mathrm{d} \sigma_{x} \mathrm{~d} \sigma_{y}$.

These results have been extensively published in the past.1,2,14,16

\section{Case of Transparent Substrates}

In the case of transparent substrates, both substrate interfaces give light scattering, as illustrated in Fig. 2.

As shown in Fig. 3, the incident light is first scattered by interface 1 in reflection $\left(I_{1}^{-}\right)$and in transmission $\left(I_{1}^{+}\right)$.

If $\gamma_{1}$ is the roughness spectrum of interface 1 and $C_{1}^{-}$and $C_{1}{ }^{+}$are the factors defined in Eqs. (2) and (3) in reflection and transmission, respectively, we can write

$$
\begin{aligned}
& I_{1}^{-}=C_{1}^{-} \gamma_{1}, \\
& I_{1}^{+}=C_{1}^{+} \gamma_{1} .
\end{aligned}
$$

$I_{1}{ }^{+}$will then be reflected on interface 2 and transmitted through interface 1 before being measured in the reflected half-space. So, as seen from the detector, the contribution $I_{1}^{t}$ of the light scattered in transmission

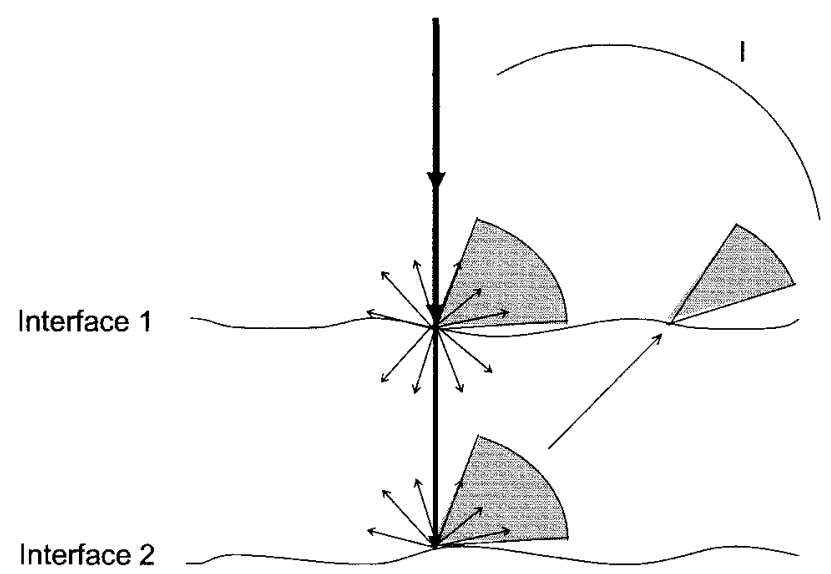

Fig. 2. Light scattering in transparent substrates.

through interface 1 is

$$
I_{1}^{t}=\mathrm{R} \beta C_{1}^{+} \gamma_{1}
$$

where $R$ is the angular reflectance and $\beta$ is the angular diffuse transmittance of the glass-air interface defined by ${ }^{6,11}$

$$
\beta=T\left(\frac{n_{0}}{n_{s}}\right)^{2} \frac{\cos \theta_{0}}{\cos \theta_{s}}
$$

On the other hand, we can consider the light transmitted through interface 1 and scattered by interface 2 (Fig. 4) with intensity $I_{2}$. If we define the incident intensity on interface 1 as $I_{0}$, taking into account the multiple reflections inside the substrate, the incident intensity on interface 2 is

$$
I_{i}=\frac{T}{1-R^{2}} I_{0}
$$

so that we have

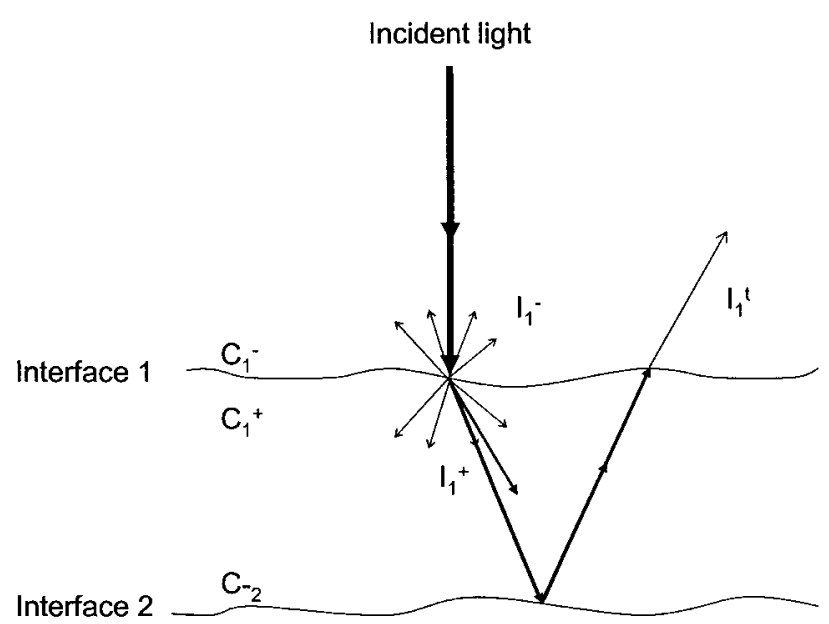

Fig. 3. Light scattered by interface 1 in a transparent substrate. 


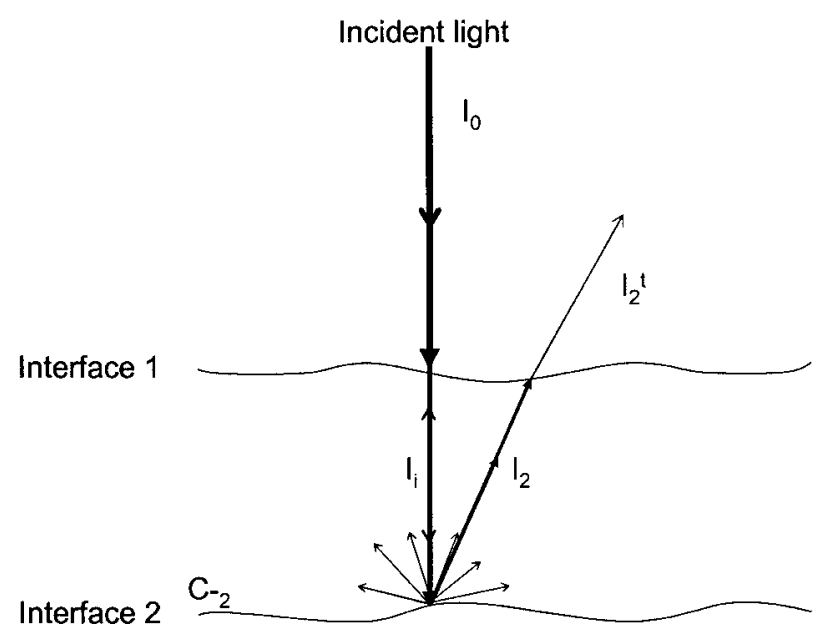

Fig. 4. Light scattered by interface 2 in a transparent substrate.

$$
I_{2}=\frac{T}{1-R^{2}} C_{2} \gamma_{2}
$$

Then $I_{2}$ is transmitted through interface 1 . So the contribution $I_{2}{ }^{t}$ of the light scattered by interface 2 is

$$
I_{2}^{t}=\beta I_{2}
$$

Finally, the scattered light coming from both interfaces and measured in the reflected half-space is given by

$$
\begin{aligned}
I(\theta) & =I_{1}^{-}(\theta)+I_{1}^{t}(\theta)+I_{2}^{t}(\theta) \\
& =C_{1}{ }^{-} \gamma_{1}+\mathrm{R}_{\beta} C_{1}{ }^{+} \gamma_{1}+\frac{T}{1-R^{2}} \beta C_{2}^{-} \gamma_{2} .
\end{aligned}
$$

If the sample is studied in the same way as an opaque sample with a single interface reachable, a roughness spectrum $\gamma$ can be deduced as follows:

$$
\gamma(\theta)=\frac{I(\theta)}{C_{1}^{-}(\theta)}
$$

where $C^{-}(\theta)=C_{1}^{-}(\theta)$.

So the equivalent roughness spectrum $\gamma_{\mathrm{eq}}$ deduced from the $I$ measurement for interface 1 is in fact

$$
\begin{aligned}
\gamma_{\mathrm{eq}} & =\frac{I(\theta)}{C_{1}^{-}(\theta)} \\
& =\frac{I_{1}^{-}(\theta)+I_{1}^{t}(\theta)+\beta I_{2}(\theta)}{C(\theta)} \\
& =\gamma_{1}+R \beta \frac{C_{1}^{+}(\theta)}{C_{1}^{-}(\theta)} \gamma_{1}+\frac{T}{1-R^{2}} \beta \frac{C_{2}^{-}(\theta)}{C_{1}^{-}(\theta)} \gamma_{2} .
\end{aligned}
$$

So we can see that the roughness spectrum $\gamma$ deduced for interface 1 is different from both $\gamma_{1}$ and $\gamma_{2}$ and that it is not an obvious combination of $\gamma_{1}$ and $\gamma_{2}$. In Fig. 5 the equivalent spectrum $\gamma_{\mathrm{eq}}$ is plotted for two spectra simulating $\gamma_{1}$ and $\gamma_{2}$ for the case in which $\gamma_{1}=\gamma_{2}$. This shows that in the case of transparent substrates, the spectrum deduced from a classical measurement contains an error due to the secondinterface contribution that is not negligible.

So, in the case of transparent substrates, to provide an adequate sample characterization, one must discriminate between the light scattered by both interfaces. Several solutions were proposed a few years ago ${ }^{17}$ with limited success, based on simultaneous data analysis in the reflected and transmitted halfspaces. We propose here a simple solution based on geometrical considerations, which permits the isolation of the scattering from each face.

\section{Experimental Setup and Theoretical Investigation}

The measurements were carried out on a scatterometer developed at the Fresnel Institute and were

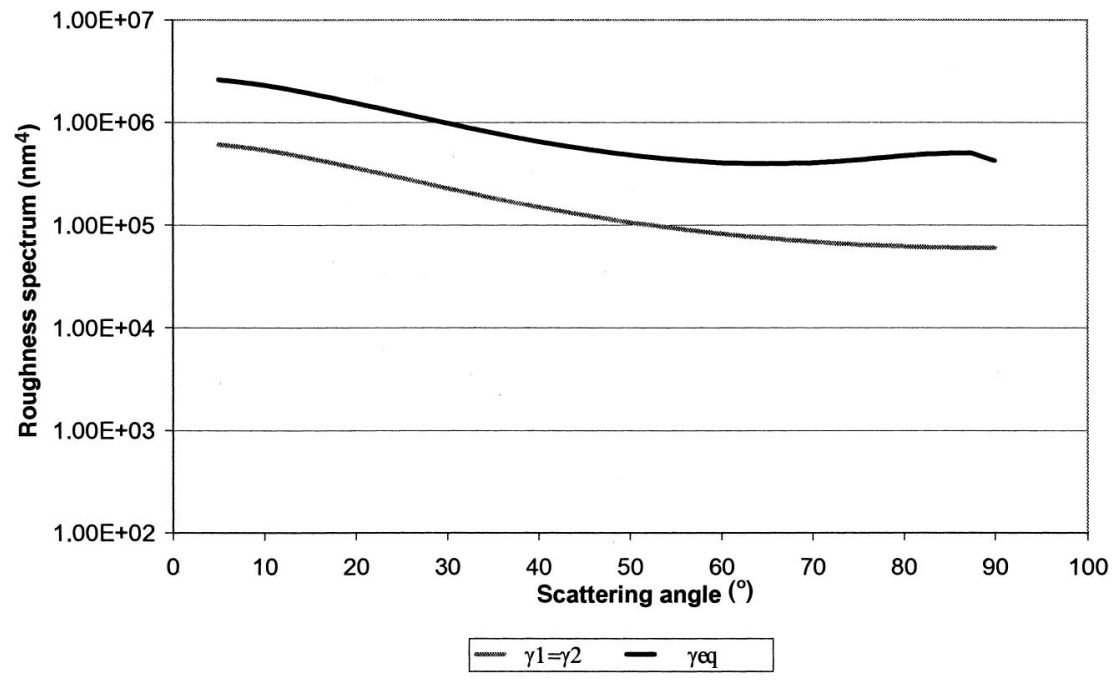

Fig. 5. Difference between real and equivalent spectra. 


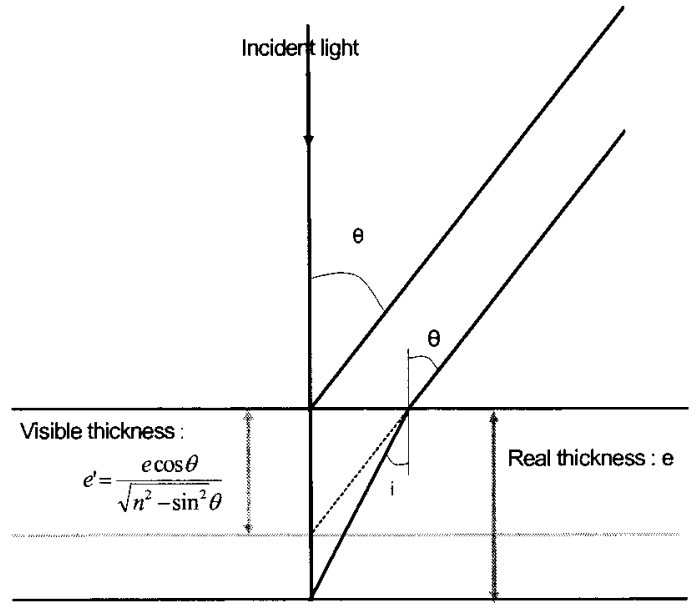

(a)

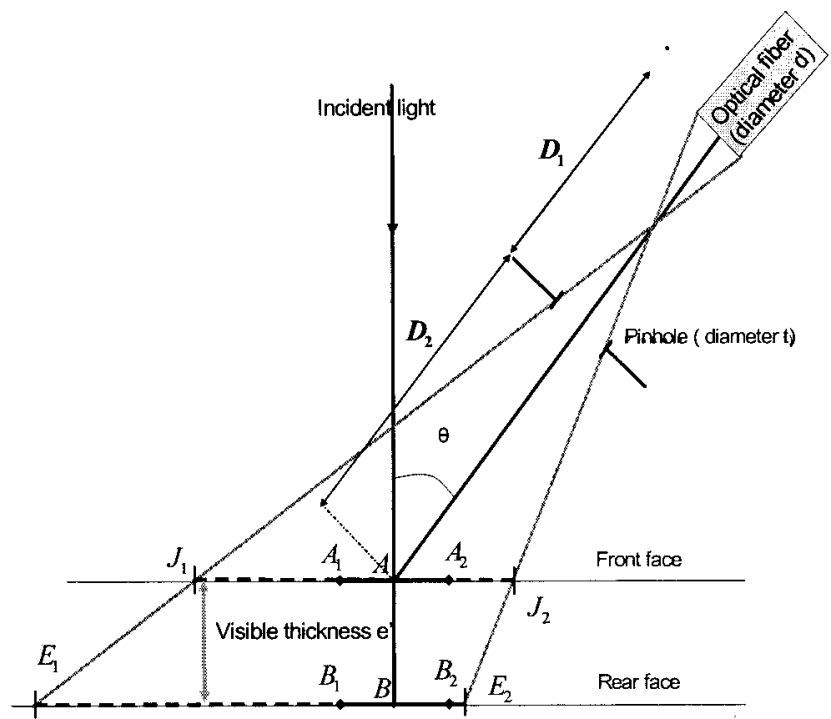

(b)

Fig. 6. Side view showing the separation of both faces using the geometrical approach. (a) Definition of the equivalent thickness of the sample as a function of the scattering angle. (b) Visualization of the areas illuminated and measured by the scatterometer on each interface of the sample.

based on the collection in the far field of scattered light by a detector placed on a rotating arm. ${ }^{15}$ We now describe those modifications realized to perform the present study.

An elementary optical system was added on the rotating arm, between the sample and the collecting optical fiber. This optical system is defined in the simplest way by the fixation of a pinhole in the direction of the measured direction $\theta$, as illustrated in Fig. 6 . The eligible parameters are $D_{1}$ and $D_{2}$, the distance from the hole to the sample and from the hole to the optical fiber, respectively, with $t$ the diameter of the hole. Adjustment of these parameters allows interface discrimination optimization.

We can see in Fig. 7 that using this optical system allows an area $S_{1}$ to be fully captured by the scatterometer on the front interface of the sample, and in the

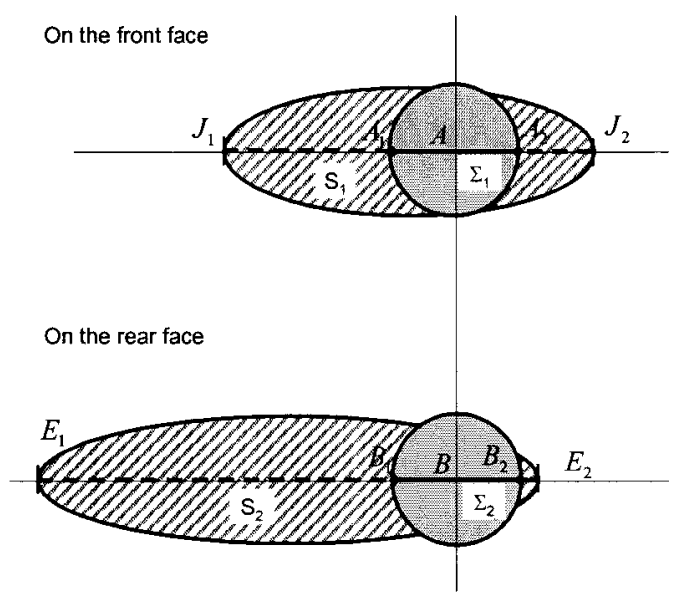

Fig. 7. Top view showing the separation of both faces using the geometrical approach. same way, an area $S_{2}$ can be fully captured by the captor on the rear sample interface. $S_{1}$ and $S_{2}$ can be defined as a function of parameters $t, D_{1}$, and $D_{2}$. In other words, the optical fiber can collect only light scattering that originates from $S_{1}$ and $S_{2}$. These surfaces are ellipsoidal, and their areas vary with angle $\theta$. Therefore all parameters must be chosen to reduce or increase the ratio $S_{1} / S_{2}$, so that one surface can be measured regardless of the other. To go further we define the capture ratio by

$$
C=\frac{S_{2} \cap \sum_{2}(\text { face } 2)}{S_{1} \cap \sum_{1}(\text { face } 1)},
$$

where $\sum_{1}$ and $\sum_{2}$ are the illuminated areas on each interface, $\left(S_{1}\right.$ and $\left.S_{2}\right)$ are the areas measured by the captor on each interface, and $S_{1} \cap \sum_{1}$ is the area common between $S_{1}$ and $\sum_{1}$. This ratio permits the

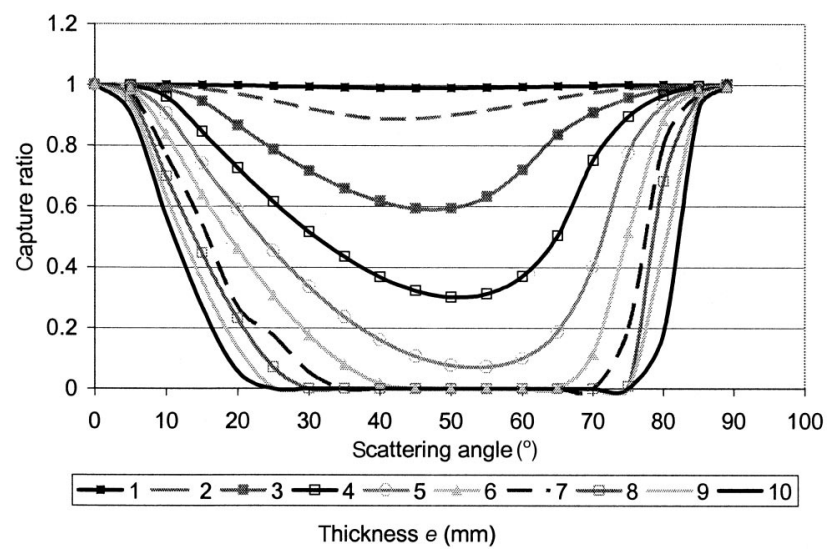

Fig. 8. Capture ratio for different sample thicknesses. 


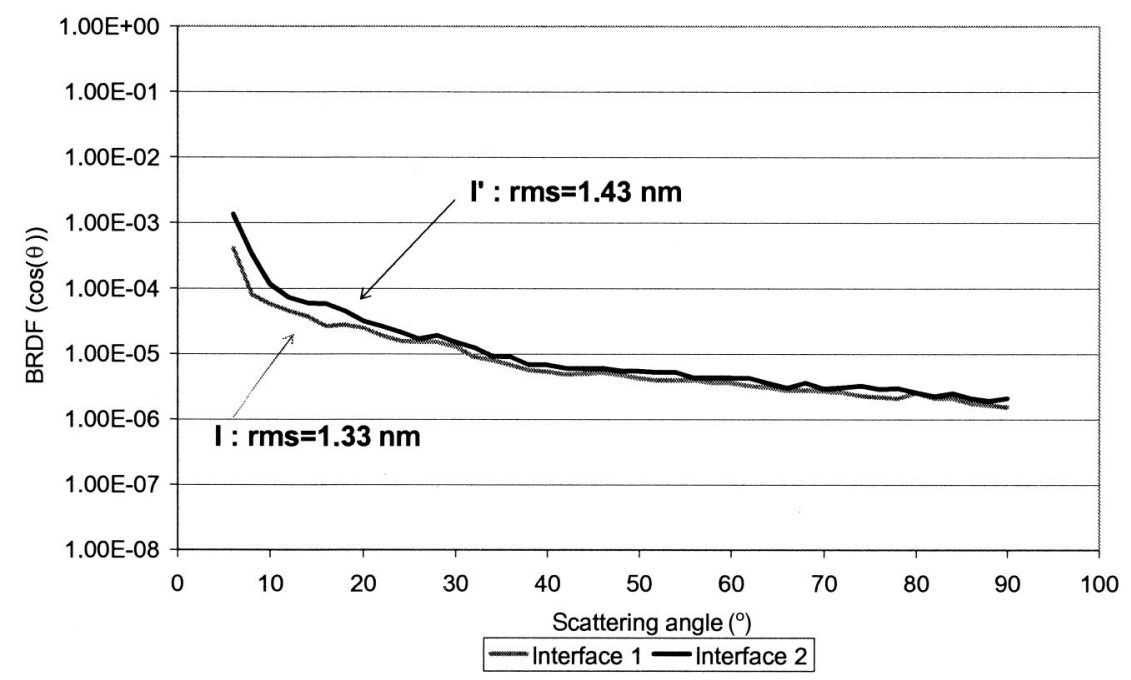

Fig. 9. Intensity scattered $I$ by a superpolished transparent substrate measured without the separation system. BRDF, bidirectional reflectance distribution function.

quantification of the amount of scattering from interface 2, which is simultaneously collected with the scattering from interface 1. All parameters in Fig. 6 can be calculated by trigonometric relations and numerical implementations. The visible areas depend on the thickness sample and, more precisely, on the equivalent thickness $e^{\prime}$, which is

$$
e^{\prime}=\frac{e \cos \theta}{\left(n^{2}-\sin ^{2} \theta\right)^{1 / 2}}
$$

In Fig. 8 the capture ratio is numerically calculated as a function of the scattering angle $\theta$ for different values of the sample thickness. These results were obtained with $D_{1}=D_{2}=20 \mathrm{~cm}$ and $t=1 \mathrm{~mm}$.

It must be noticed that the capture ratio equals zero in a limited angular domain, provided that the sample is thick enough. In this case the front inter- face can be insulated. The same technique can then be used to characterize the other surface (rotation of sample perpendicular to its plane).

\section{Application}

To validate the technique, we used $9 \mathrm{~mm}$ thick optical polish-quality fused-silica samples. Light-scattering measurements were performed first without the separation system (Fig. 9) and then with the separation system (Fig. 10). In this last measurement configuration, when the incident light arrives on interface 1 , we measure $I_{1}$. Then the sample is turned and illuminated on interface 2 , and $I_{2}{ }^{\prime}$ is measured.

On the one hand, we can see a difference in the scattering level, depending on whether the separation system is used. On the other hand, we note that there is a higher intensity gap between the two measurements when the separation system is used.

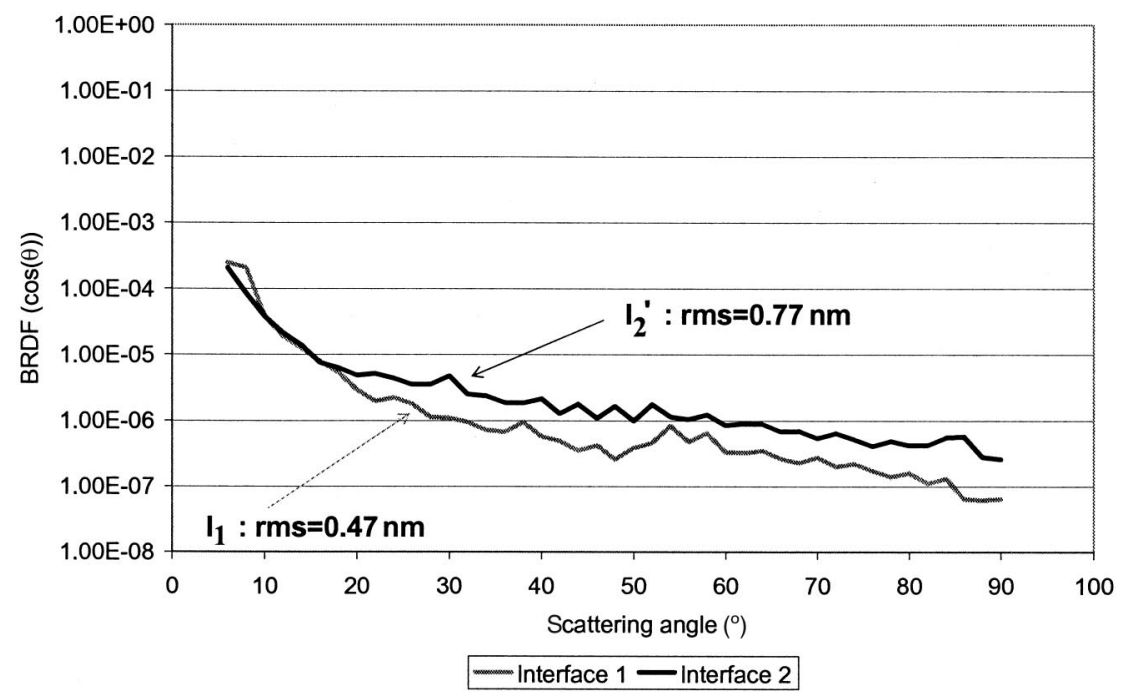

Fig. 10. Intensity scattered $I_{1}$ and $I_{2}$ by each interface of a superpolished transparent substrate measured with the separation system. 


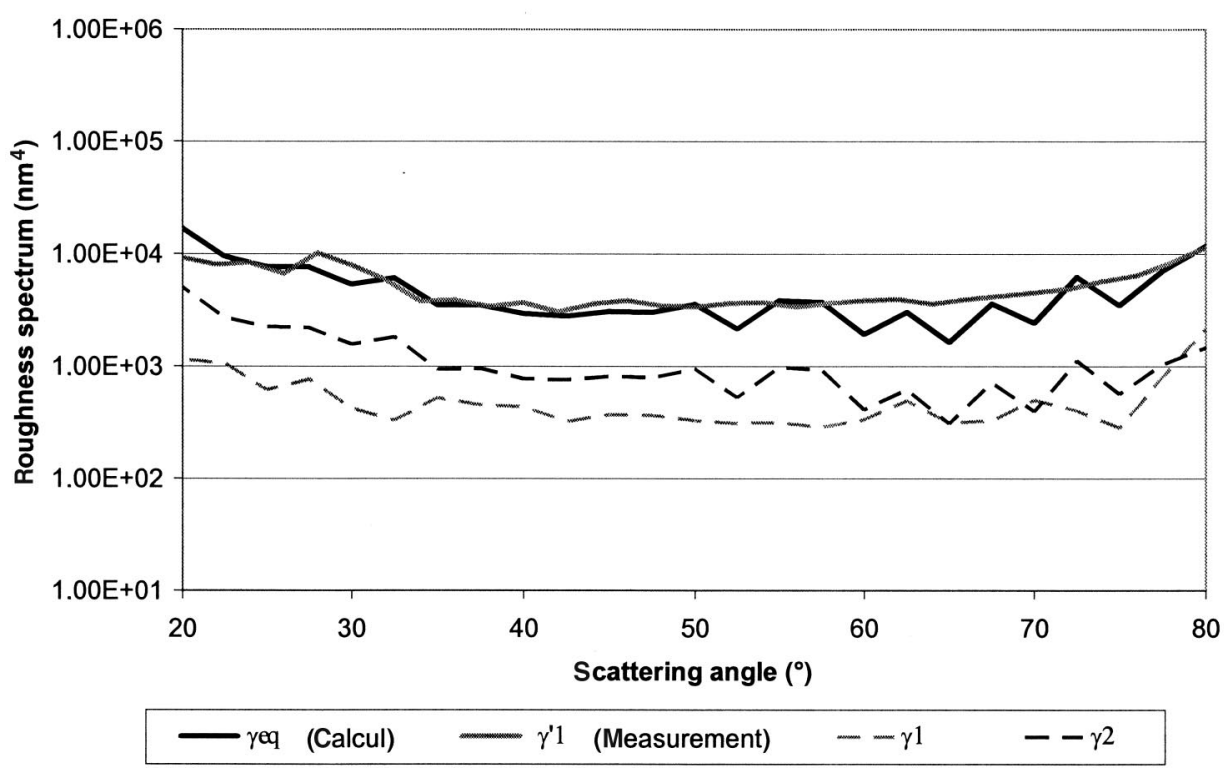

Fig. 11. Calculated and measured roughness spectra (higher spectra values for higher scattering angles are due to the parasite light coming from the sample side).

Let us now see whether the experiment confirms what we saw in Section 4 . The roughness spectra $\gamma_{1}$ and $\gamma_{2}$ of interfaces 1 and 2, respectively, were calculated with Eq. (1) from the intensities $I_{1}$ and $I_{2}{ }^{\prime}$, respectively, given in Fig. 10 and measured with the separation system. The spectrum $\gamma_{1}{ }^{\prime}$ of interface 1 was deduced from the intensity $I$ measured without the separation system and shown in Fig. 9. In addition, the equivalent roughness spectrum $\gamma_{\text {eq }}$ of interface 1, calculated with Eq. (17), which is expected knowing $\gamma_{1}$ and $\gamma_{2}$, is plotted in Fig. 11. First, we can see that $\gamma_{1}$ and $\gamma_{2}$ are different from each other and that $\gamma_{1}$ and $\gamma_{2}$ are lower than $\gamma_{1}{ }^{\prime}$, confirming the results given in Figs. 9 and 10 .

Moreover, we notice that the calculated spectrum $\gamma_{\text {eq }}$ has the same level as that of the measured spectrum $\gamma_{1}{ }^{\prime}$. We can then deduce that the $\gamma_{1}$ and $\gamma_{2}$ spectra are the real spectra of interfaces 1 and 2 , respectively.

To finish, we can quantify the roughness values of each interface and compare them with the values that should be obtained in the absence of the separation system. Results are shown in Figs. 9 and 10. In Fig. 9 the two curves correspond to the measurement of the same sample insulated on one interface and then on the other. The curves are nearly the same, with a roughness value close to $1.4 \mathrm{~nm}$. In this case both interfaces are contributing to light scattering, and the result is nearly the same regardless of the illuminated interface. In Fig. 10 we can see that the separation system permits the measurement of the differences between both faces. In this case the roughnesses deduced are $0.47 \mathrm{~nm}$ for interface 1 and $0.77 \mathrm{~nm}$ for interface 2 .

\section{Comparison with Atomic Force Microscopy Measurements}

The final verifications were performed by atomic force microscopy (AFM). Previous studies ${ }^{14,16,18}$ have shown that it is possible to predict angular light scattering through the measurement of the surface profile by using microscopic techniques. Toward this end, we use AFM. Equation (1) can be applied. The optical term $C$ is calculated by using the optical parameters (index, wavelength, and polarization),1,2 and the roughness spectrum is given by Eq. (6), where the profile $h$ comes from the microscopic measurement. We can see in Fig. 12 the AFM image of another $9 \mathrm{~mm}$ thick optical polish-quality fused-silica sample.

However the AFM bandpass $\left(B_{\mathrm{AFM}}\right)$ must be adjusted to the ARS one $\left(B_{\mathrm{ARS}}\right)$, since we have

- $B_{\mathrm{ARS}}(\lambda)=\left[\sin \theta_{m} / \lambda, 1 / \lambda\right]$ with $\lambda$ the incident wavelength,

- $B_{\mathrm{AFM}}(\Delta x)=[1 / L, 1 / 2 \Delta x]$ with $L^{2}$ the measured area and $\Delta x$ the sampling interval.

The parameters we chose are $L=50 \mu \mathrm{m}$ and $N$ $=256$, which guarantee an intersection of ARS and AFM bandwidths. ${ }^{16,19}$ Therefore the AFM data can be

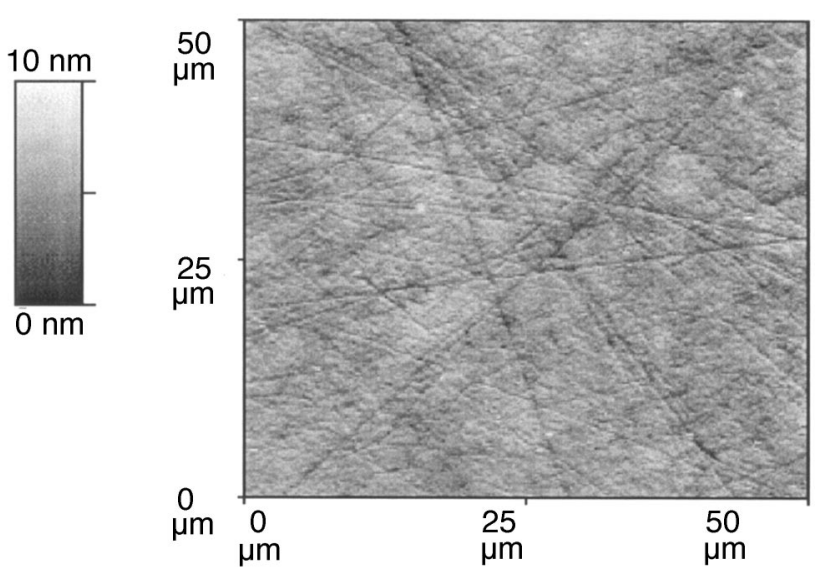

Fig. 12. AFM image of the sample surface. 


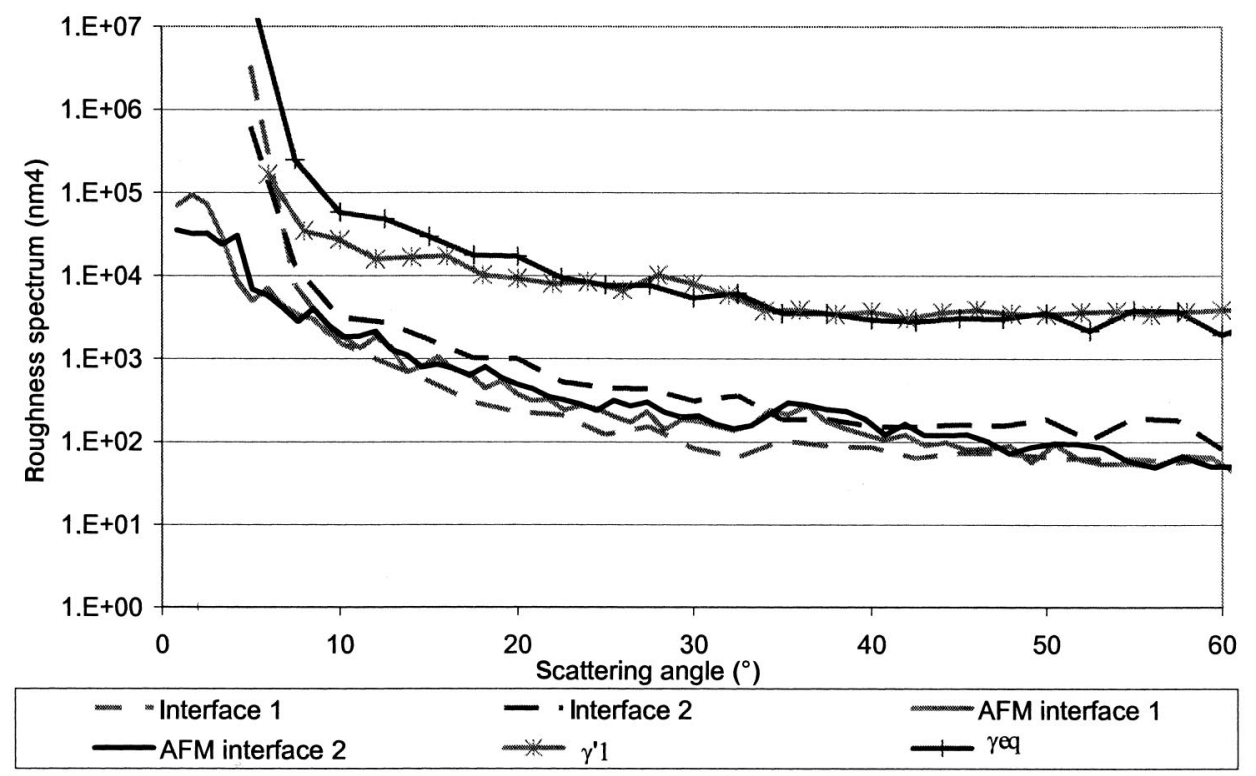

Fig. 13. Comparison between the roughness spectra calculated from the AFM measurements of each interface of the sample and the measured spectra for each interface.

used to calculate the roughness spectrum, and this spectrum is then used in the scattering calculation.

In Fig. 13 we can see that the angular scattering collected from the front and back surfaces is in agreement with the AFM calculation for the same surfaces. Differences still remain but are due to the stationarity of the surfaces at the AFM scale. On the other hand, the measurement performed without the separation system (Fig. 13) yields a roughness spectrum that is very far from the real spectrum.

\section{Conclusion}

A technique to insulate scattering from each interface of a transparent substrate has been introduced. Such a procedure allows us to characterize the micropolish of each face of a superpolished transparent sample. The results were checked with AFM measurements.

This research was partially supported by the European Union within the framework of the Cooperative Research Action for Technology, Development and Application of Compact Mode-Locked Lasers project.

\section{References}

1. C. Amra, "Light scattering from multilayer optics. Part A: Investigation tools," J. Opt. Soc. Am. A 11, 197-210 (1994).

2. C. Amra, "Light scattering from multilayer optics. Part B: Application to experiment," J. Opt. Soc. Am. A 11, 211-226 (1994).

3. J. M. Bennet and L. Mattsson, Introduction to Surface Roughness and Scattering (Optical Society of America, 1989).

4. L. Bruel, "Etude comparative de méthodes optiques et mécanique pour caractériser les états de surface. Diffusion de la lumière par les rugosités de surface et inhomogénéités de volume dans les empilements de couches minces," Ph.D. dissertation (Univ Aix-Marseille III, Marseille, France, 1992).

5. P. Croce and L. Prod'homme, "Ecarts observés dans l'interprétation des indicatrices de diffusion optique par des théories vectorielles simples," J. Opt., (Paris) 16, 143-151 1985.

6. J. M. Elson, J. P. Rhan, and J. M. Bennet, "Relationship of the total integrated scattering from multilayer-coated optics to angle of incidence, polarization, correlation-length, and roughness cross-correlation properties," Appl. Opt. 22, 3207-3219 (1983).

7. A. Sentenac and J. J. Greffet, "Mean-field theory of light scattering by one-dimensional rough surfaces," J. Opt. Soc. Am. A 15, 528-532 (1998).

8. A. Sentenac, J. J. Greffet, H. Giovannini, and M. Saillard, "Scattering from rough inhomogeneous media: splitting of surface and volume scattering," J. Opt. Soc. Am. A 19, 727-736 (2002).

9. P. Vincent, "Differential method," in Electromagnetic Theory of Gratings, Vol. 22 of Topics in Current Physics (SpringerVerlag, 1980), pp. 101-121.

10. A. G. Voronovitch, "Small slope approximation in wave scattering from rough surfaces," Sov. Phys. JETP 1, 65-70 (1985).

11. D. Torricini and C. Amra, "Light scattering to characterize both faces of transparent substrates: embedded and radiative transfer," in Optical Interference Coatings Proc. SPIE 2253, 1117-1130 (1994).

12. M. Zerrad, C. Deumié, and M. Lequime, "Diffusion de la lumière par des substrats transparents: caractérisation d'états de surfaces," poster presented at the Horizons de l'Optique, Toulouse, France, 3-5 September 2003.

13. C. Amra, "First order vector theory of bulk scattering in optical multilayers," J. Opt. Soc. Am. A 10, 365-374 (1993).

14. C. Amra, D. Torricini, and P. Roche, "Multiwavelength (0.45$10.6 \mu \mathrm{m})$ angle-resolved scatterometer or how to extend the optical window," Appl. Opt. 32, 5462-5474 (1993).

15. C. Deumié, "Ellipsométrie sur champ diffus et analyse multiéchelle de la microstructure des multicouches optiques: diffusion lumineuse, microscopie à force atomique, microscopie à effet tunnel optique," Ph.D. dissertation (Université AixMarseille III, Marseille, France, 1997).

16. C. Deumié, R. Richier, P. Dumas, and C. Amra, "Multiscale roughness in optical multilayers: atomic force microscopy and light scattering," Appl. Opt. 35, 5583-5594 (1996). 
17. D. Torricini and C. Amra, "Light scattering to characterize both faces of transparent substrates: radiative and embedded light," in Optical Interference Coatings Proc. SPIE 2253, 11171130 (1994).

18. P. Dumas, B. Bouffakhredine, C. Amra, O. Vatel, E. André, R. Galindo, and F. Salvan, "Quantitative microroughness using near field microscopies and optical," Europhys. Lett. 22, 717722 (1993).

19. C. Amra, C. Deumié, D. Torricini, P. Roche, and R. Galindo, "Overlapping of roughness spectra measured in macroscopic (optical) and microscopic (AFM) bandwidths," in Optical Interference Coatings, Proc. SPIE 2253, 614-630 (1994). 